\title{
Critical surface in hot and dense QCD with the vector interaction
}

\author{
Kenji Fukushima \\ Yukawa Institute for Theoretical Physics, Kyoto University, Kyoto 606-8502, Japan
}

\begin{abstract}
We discuss the chiral phase transition of hot and dense quark matter. We illustrate that the firstorder phase transition is generally favored at high baryon density and the repulsive vector-vector interaction weakens the first-order phase transition. We use the Nambu-Jona-Lasinio model with the Polyakov loop coupling for concreteness. We locate the QCD critical surface on the quark mass plane for various values of the vector coupling constant. We find that, with increasing quark chemical potential, the first-order region in the quark mass plane could shrink for sufficiently large vector coupling. This may be a possible explanation for the recent lattice QCD results by de Forcrand and Philipsen.
\end{abstract}

PACS numbers: 12.38.Aw, 11.10.Wx, 11.30.Rd, 12.38.Gc

\section{INTRODUCTION}

On the phase diagram in the plane with the axes by temperature, density, pressure, concentration, external fields, etc, the "critical point" commonly refers to the terminal point of the phase transition boundary of first order. This point has an exact second-order phase transition regardless of symmetry properties.

Interestingly enough, the existence of the critical point is a possibility not only in condensed matter physics but also in QCD (Quantum Chromodynamics) physics [1]. The QCD phase diagram with the temperature $T$ and the quark chemical potential $\mu$ has taken on significance in the application to the heavy-ion collisions at various energies as well as to the neutron star structure. There seems to be a consensus that the QCD phase transitions associated with chiral symmetry restoration and color deconfinement are smooth crossover when $T$ goes up with $\mu \simeq 0$. In contrast, the situation changes in the different regime where $\mu$ grows with $T \simeq 0$. Model studies [2, 3] suggest that the first-order chiral phase transition should occur at some $\mu$ comparable to the constituent quark mass (or one third of the baryon mass) when $T \simeq 0$. To be consistent with crossover at $T \neq 0$ and $\mu \simeq 0$, therefore, the critical point is expected to exist at intermediate $T_{E}$ and $\mu_{E}$.

It is, however, important to remark that the existence of the QCD critical point is still under dispute [4]. One interesting negative observation against the QCD critical point has come from the Monte-Carlo simulation of QCD on the lattice with varying the light-quark mass $m_{u d}$ and the strange-quark mass $m_{s}$ [5], though some other lattice simulations are rather affirmative. (For a review see Ref. [6]).

For the symmetry reason the chiral phase transition in three-flavor quark matter at $m_{u d}=m_{s}=0$ is presumably first order 7]. Because the mass term explicitly breaks chiral symmetry, the first-order transition turns to crossover at some $m_{u d}$ and $m_{s}$ [8, 9], which defines the critical boundary in the $m_{u d}-m_{s}$ plane. Model studies that support the QCD critical point predict that the first-order region in the $m_{u d}-m_{s}$ plane expands by the effect of increasing $\mu$, so that the physical quark mass point hits the critical surface at $\mu=\mu_{E}$ [10, 11]. However, de Forcrand and Philipsen [5] recently claim that the first-order region should not expand but rather shrink at higher density as long as $\mu / T$ is small.

The purpose of this paper is twofold:

1) We will extract the general feature of the quasiparticle description at high density to favor the first-order phase transition. We make use of simple closed expressions by limiting our discussions to the $T=0$ case. In fact, the confirmation of the first-order phase transition at $T=0$ and $\mu \neq 0$ suffices for the existence of the QCD critical point since the QCD phase transition at $\mu=0$ and $T \neq 0$ is crossover. In the same way we discuss the effect of the repulsive vector-vector interaction on the general ground. These are all discussed in Sec. [II

2) We will point out in Sec. [II] that the shrinkage of the first-order region in the $m_{u d}-m_{s}$ plane is not uncommon once we take account of a vector-vector interaction. We draw the critical surface using the Polyakov loop augmented Nambu-Jona-Lasinio (PNJL) model to exemplify the effect of the vector interaction, which may be a likely explanation for the results by de Forcrand and Philipsen.

\section{FIRST-ORDER PHASE TRANSITION}

Let us first consider a simple chiral model at $T=0$ with and without the vector interaction. We will do so because we should understand the underlying mechanism for possibility of the first-order phase transition at $T=0$ and $\mu \neq 0$ to elucidate the effect of the vector interaction.

The simplest case in the chiral limit is enough for our present demonstration in which one dynamical mass $M$ serves as the order parameter. Here $M$ is either the constituent quark mass in the color deconfined phase or one third of the nucleon mass minus binding energy of nuclear matter if the system confines color. Although we can formulate both, we shall focus on the former, i.e. deconfined quark matter, hereafter. (For the latter, see discussions in Ref. [3].) We assume that the pressure $P_{\chi}[M]$ results in the chiral symmetry broken phase at zero density. That is, the free energy, $-P_{\chi}[M]$, has minima lo- 
cated at $M= \pm M_{0}$; we simply postulate the following form;

$$
P_{\chi}[M]=-a\left(M_{0}^{2}-M^{2}\right)^{2}
$$

with a parameter $a$. Here we note that a linear term in $M$ should be present if the current quark mass is nonzero. We can neglect this explicit chiral symmetry breaking in the qualitative level because such a term has only minor effects on the phase transition in the two-flavor sector. In the three-flavor case, in contrast, the $\mathrm{U}_{\mathrm{A}}(1)$ breaking term generates a cubic term in $M$ which favors the first-order phase transition. We will not think of this situation; our purpose here is to see how the first-order transition is possible at high density even though it is of second order at vanishing density. Thus, the above form of Eq. (1) is valid when all the quarks are massless and the three-flavor $\mathrm{U}_{\mathrm{A}}(1)$ breaking is not significant.

Now let us turn finite $\mu$ on. As long as $\mu$ is smaller than the lowest-lying mass of fermionic excitation, nothing happens and the vacuum remains empty. Once $\mu$ exceeds a certain threshold $M$, a finite amount of density appears. The pressure has a contribution from the density which is generally expressed as

$$
P_{\mu}[M]=\int_{0}^{\mu} d \mu^{\prime} n_{q}\left(\mu^{\prime}\right)
$$

Here $n_{q}(\mu)$ represents the fermion density. In the quasiparticle picture it is given by the integrated Dirac-Fermi distribution function with the constituent mass;

$$
\begin{aligned}
n_{q}(\mu) & =\nu \int \frac{d^{3} p}{(2 \pi)^{3}}\left[\frac{1}{e^{(\varepsilon-\mu) / T}+1}-\frac{1}{e^{(\varepsilon+\mu) / T}+1}\right] \\
& \stackrel{T=0}{\longrightarrow} \frac{\nu}{6 \pi^{2}}\left(\mu^{2}-M^{2}\right)^{3 / 2} \theta\left(\mu^{2}-M^{2}\right)
\end{aligned}
$$

where $\varepsilon=\sqrt{p^{2}+M^{2}}$ and $\nu$ is the fermionic degrees of freedom (color $\times$ flavor $\times$ spin $)$. In two-flavor quark matter, for relevant example, $\nu=(3$ colors $) \times(2$ flavors $) \times$ $(2$ spins $)=12$. We note that $\theta$ denotes the Heaviside theta function, which signifies that the system at $\mu<M$ is empty. In fact, the theta function is essential to make a double-peak shape in the total pressure, as we will see soon.

It is possible to perform the integration (2) to find an analytical expression with logarithmic terms. To simplify our qualitative analysis, however, we shall introduce an approximation as

$$
P_{\mu}[M] \approx \frac{\nu}{24 \pi^{2} \mu^{2}}\left(\mu^{2}-M^{2}\right)^{3} \theta\left(\mu^{2}-M^{2}\right),
$$

which turns out to be a good approximation as shown in Fig. 1. The solid curve represents Eq. (4), while the dotted curve is Eq. (2) with Eq. (3) substituted. Because more particles can reside in the Fermi sphere for smaller mass, $P_{\mu}[M]$ has a maximum at $M=0$.

Let us consider the condition for $P[M]=P_{\chi}[M]+$ $P_{\mu}[M]$ to have a first-order phase transition. Here $P_{\chi}[M]$

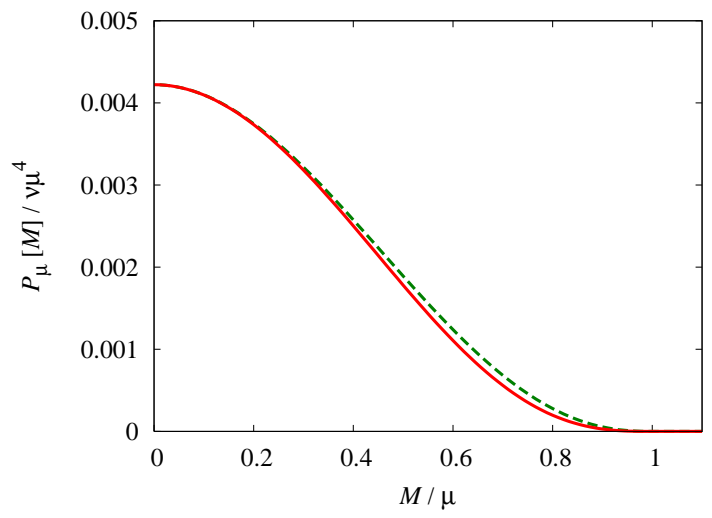

FIG. 1: Comparison between the exact integration in Eq. (2) (by the dotted curve) and the approximation in Eq. (4) (by the solid curve).

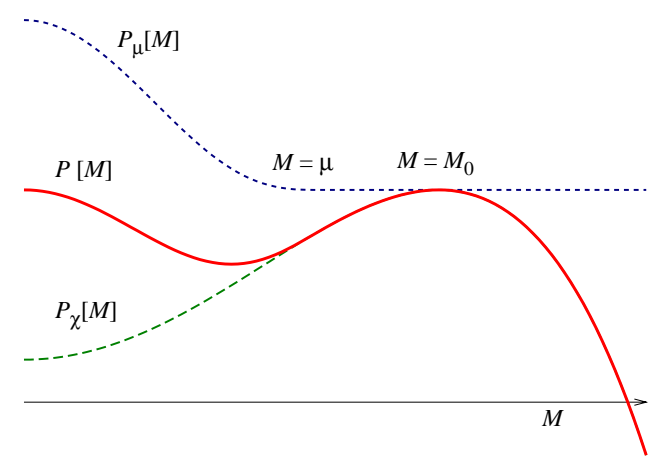

FIG. 2: Sketch of the double-peak pressure $P[M]$ resulting from the sum of $P_{\chi}[M]$ and $P_{\mu}[M]$.

and $P_{\mu}[M]$ have a peak at $M=M_{0}$ and $M=0$ respectively (see Fig. 2). The existence of double peaks in $P[M]$ requires that $\mu \lesssim M_{0}$, meaning that $\mu$ should not be much greater than $M_{0}$. [So, $\mu$ can be larger than $M_{0}$ slightly.] This is necessary for the peak at $M=M_{0}$ to survive. At $M=0$ the pressure curvature (i.e. the coefficient of the $M^{2}$ term) should be negative, that is;

$$
a<\frac{\nu}{16 \pi^{2}} \frac{\mu^{2}}{M_{0}^{2}} \lesssim \frac{\nu}{16 \pi^{2}} .
$$

At the first-order critical point the peak at $M=0$ is as high as the second peak at $M=M_{0}$ (neglecting a small shift by the contribution from $P_{\mu}[M]$ ), which yields the critical condition that

$$
a \simeq \frac{\nu}{24 \pi^{2}} \frac{\mu_{c}^{4}}{M_{0}^{4}} .
$$

As long as $\mu$ is raised with $\mu \lesssim M_{0}$ satisfied, the curvature condition (5) is sufficient for the existence of $\mu_{c}$ deduced from Eq. (6). This is another way to see why we should have required $\mu \lesssim M_{0}$.

We shall next take account of the mean-fields from the vector-vector interaction, $-G_{V}\left(\bar{\psi} \gamma_{\mu} \psi\right)\left(\bar{\psi} \gamma^{\mu} \psi\right)$ 11, 
12, 13, 14]. Because $\left\langle\bar{\psi} \gamma^{0} \psi\right\rangle$ is nothing but the fermionic density, roughly speaking, the vector interaction generates a contribution to the pressure;

$$
P_{V}[M]=-G_{V} n_{q}^{2}=-\frac{G_{V} \nu^{2}}{36 \pi^{4}}\left(\mu^{2}-M^{2}\right)^{3} \theta\left(\mu^{2}-M^{2}\right)
$$

at $T=0$, which takes the same functional form as the approximated (4). [In the above we have constructed $P_{V}[M]$ using $n_{q}$ given by $\partial P_{\mu} / \partial \mu$. This approximation is qualitatively reasonable, but not self-consistent with the full pressure from which $n_{q}$ should have been inferred. We will come back to this point later.] The coefficient in Eq. (4) is hence modified by the effect of Eq. (7) (that is, the effective fermionic degrees of freedom are reduced), and the curvature condition is then

$$
a<\left(1-\frac{2 \nu G_{V} \mu^{2}}{3 \pi^{2}}\right) \frac{\nu}{16 \pi^{2}} \frac{\mu^{2}}{M_{0}^{2}} .
$$

Therefore, even though we start with $a$ that satisfies Eq. (5), there is a critical $G_{V}$ for which Eq. (8) is not satisfied within $\mu \lesssim M_{0}$. Then, the first-order phase transition would disappear.

Let us see concrete numbers; in the conventional NJL model with two flavors [15], for which our discussions based on Eq. (11) are valid, one can read $a$ as

$$
a=\frac{1}{2 M_{0}^{2}}\left(\frac{\nu \Lambda^{2}}{8 \pi^{2}}-\frac{1}{4 G_{S}}\right)=0.067,
$$

where we used $\nu=12$ and the ultraviolet cutoff $\Lambda=$ $631 \mathrm{MeV}$, the four-fermion coupling $G_{S} \Lambda^{2}=2.19$, and the resultant $M_{0}=336.2 \mathrm{MeV}$ [15]. The first term originates from the zero-point energy and the second from the four-fermion interaction. Then, the right-hand side of Eq. (5) is 0.076 and the inequality is certainly satisfied. In the case of the chiral quark model [16], for comparison, $a$ is estimated as $a \simeq m_{\sigma}^{2} f_{\pi}^{2} /\left(8 M_{0}^{4}\right)$, which yields $a=0.02 \sim 0.05$ depending on the value of the $\sigma$ meson mass. This is again within the region of the first-order phase transition.

The critical point in the NJL model case is

$$
\mu_{c}=1.07 M_{0},
$$

from Eq. (6). This estimate is consistent with the empirical value in the NJL model study.

It is easy to see the effect of $G_{V}$ from Eq. (8). As we have confirmed the critical $\mu$ is nearly $M_{0}$ and we can replace $\mu \rightarrow M_{0}$ in Eq. (8) approximately. Then, the inequality does not hold when

$$
G_{V}>0.25 G_{S}
$$

meaning that such $G_{V}$ makes the first-order phase transition disappear at all along the $\mu$ axis. It is impressing that this rough estimate is fairly consistent with a more serious analysis in the NJL model [12].

We can learn from the above argument that the repulsive vector-vector interaction reduces the pressure arising

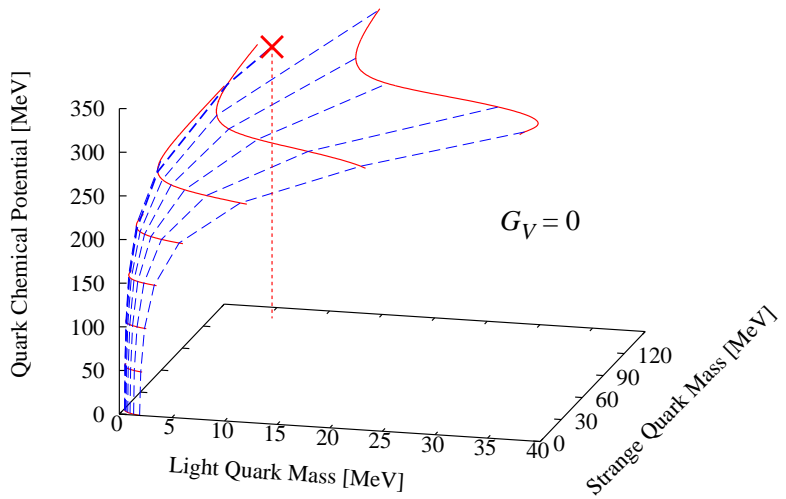

FIG. 3: Critical surface with standard curvature in the PNJL model without the vector interaction. The physical mass point in this model is $\left(m_{u d}=5.5 \mathrm{MeV}, m_{s}=135.7 \mathrm{MeV}\right)$ which hits the critical surface at $\mu=\mu_{E}$.

from the degenerated particles in the Fermi sphere. To achieve chiral restoration with such strong vector interaction, then, $\mu>M_{0}$ is necessary and a peak around $M=M_{0}$ is washed away by the tail in $P_{\mu}[M]+P_{V}[M]$ which extends up to $M=\mu$. This is the qualitative mechanism how the vector interaction would hinder the first-order phase transition.

\section{THREE-FLAVOR MASS PLANE}

From the point of view of thermodynamic pressure, so far, we have seen how the first-order phase transition could occur at $\mu=\mu_{c} \simeq M_{0}$ especially in the case of two-flavor cold quark matter.

Let us now proceed to the main part of our discussions on three-flavor quark matter. For a fixed value of $\mu$, the chiral phase transition at finite $T$ is of first order when the current quark masses $m_{u d}$ and $m_{s}$ stay small, and becomes of crossover if $m_{u d}$ and $m_{s}$ exceed a critical boundary. Therefore, in the $3 \mathrm{D}$ space of $m_{u d}, m_{s}$, and $\mu$, the critical condition makes a hypersurface. For concreteness we adopt the PNJL model used in Ref. [11] to draw the critical surface. The NJL model might allow for artificial quark excitations at finite temperature due to the lack of confinement. This tends to shift the location of the critical point $\left(\mu_{E}, T_{E}\right)$ to higher $\mu_{E}$ and lower $T_{E}$ because the temperature effect smears the firstorder phase transition induced by density. The PNJL model cures this (for instance $T_{E}$ changes $\sim 50 \mathrm{MeV}$ in the NJL model to $\sim 100 \mathrm{MeV}$ in the PNJL model with the same parameter set [17]) by means of the color projection by the Polyakov loop which is a colored phase factor associated with single-quark excitation. Since the model details are not our main focus, we simply refer to Ref. [11, 17] for details.

Figure 3 is the critical surface with standard curvature 
obtained in the PNJL model. Above the critical surface the finite- $T$ phase transition is of first order, while no sharp phase transition takes place below the surface. The second-order phase transition sits on the critical surface. This is just one model example but shows typical behavior in model studies [10]. The physical mass point hits the critical surface at $\mu=\mu_{E}=313.5 \mathrm{MeV}$ where the critical temperature is $T=T_{E}=101.8 \mathrm{MeV}$ (shown by a cross in Fig. (3).

It is general in the quasi-particle picture that the density effect induces a pressure like Eq. (2) whose maximum is located in the (partially) chiral symmetric phase. In the presence of such an additional peak near $M_{u d} \simeq m_{u d}$, $M_{s} \simeq m_{s}$, the first-order transition region is enhanced with increasing $\mu$.

The problem is that this curvature of the critical surface as shown in Fig. 3 might be inconsistent with the lattice observation [5] even though the model results seem to be rather robust not relying on any special assumption.

We here propose one scenario that has a natural account for the lattice results and, at the same time, may not necessarily exclude the existence of the critical point. The necessary ingredient is the vector-vector interaction alone. As a matter of fact, the repulsive vector interaction is anticipated from the hadron property [12, 18]. Figures 4 and 5 are the examples from the PNJL model with the vector interaction incorporated. Here we should explain the mean-field treatment for the vector interaction in a self-consistent way $2,11,12,13$, 14, 18]. The zeroth component in the vector interaction, $-G_{V}\left(\bar{\psi} \gamma_{0} \psi\right)\left(\bar{\psi} \gamma^{0} \psi\right)$, produces the mean-field terms, $-2 G_{V} n_{q} \bar{\psi} \gamma^{0} \psi+G_{V} n_{q}^{2}$. The former term adds to the chemical potential as $\mu \rightarrow \mu-2 G_{V} n_{q}$ and the latter adjusts the larger contribution from the former. Then, the mean-field $n_{q}$ is fixed by $\partial P / \partial n_{q}=0$ which guarantees the thermodynamic relation; $n_{q}-\partial P / \partial \mu=0$. [Note that $\partial P / \partial n_{q}=-2 G_{V} \partial P / \partial \mu$.]

We have chosen a considerably large value of $G_{V}$ to make it easier to perceive the effect visually. The point is that the inclusion of $G_{V} \neq 0$ induces the opposite curvature to that in Fig. 3 at small $\mu$, and eventually the curvature returns to the standard one at larger $\mu$. It should be noted that the first-order region at $\mu=0$ does not change but the axis scale in Figs. 4 and 5 is different from Fig. 3 ,

Although our aim is just to present some demonstrations like Figs. 4 and 5 , it is intriguing to make a quantitative comparison. Along the diagonal $m=m_{u d}=m_{s}$ line, the critical mass is expanded in terms of $\mu$ as

$$
\frac{m_{c}(\mu)}{m_{c}(0)}=1+c_{2}\left(\frac{\mu}{\pi T_{c}}\right)^{2}+c_{4}\left(\frac{\mu}{\pi T_{c}}\right)^{4}+\cdots .
$$

Our calculations give $c_{2}=5.88$ and $c_{4}=43.8$ at $G_{V}=0$ as shown by the solid curve in Fig. 6. The lattice results are, on the other hand, $c_{2}=-0.7(4)$ and $c_{4} \simeq 0$ in the first paper of Ref. [5] which is indicated by the upper shaded region in Fig. 6. In the last paper of Ref. 6] significantly different values are reported; $c_{2}=-3.3(3)$ and

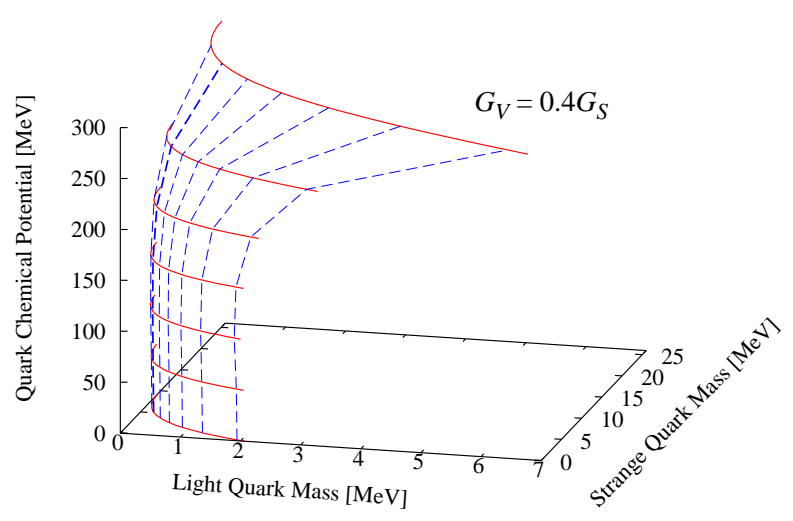

FIG. 4: Demonstration of the effect of the vector interaction in the PNJL model with $G_{V}=0.4 G_{S}$.

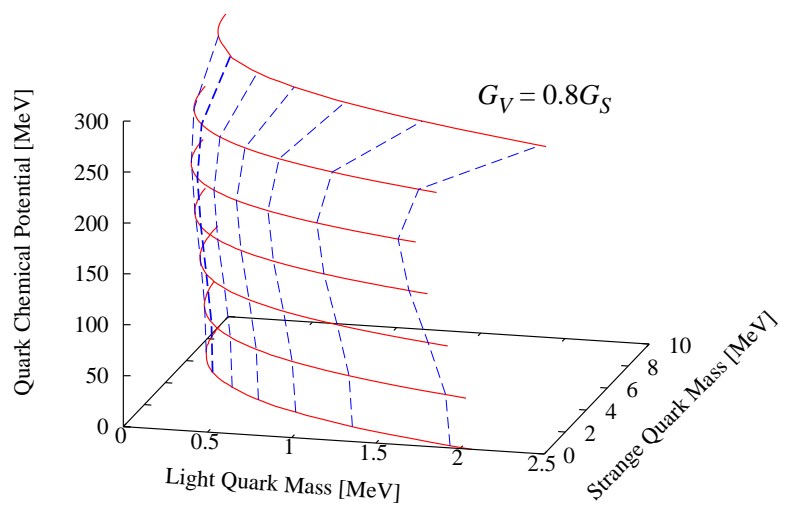

FIG. 5: Demonstration of the effect of the vector interaction in the PNJL model with $G_{V}=0.8 G_{S}$.

$c_{4}=-47(20)$ as shown by the lower shaded region. In our calculations the vector interaction drastically alters the curvature from the solid curve to the dashed (dotted) curve in Fig. 6 at $G_{V}=0.4 G_{S}\left(G_{V}=0.8 G_{S}\right)$.

From Fig. [6 we may say that some $G_{V}<0.4 G_{S}$ could be enough to understand the results in the first paper of Ref. [5], while the vector interaction alone is not sufficient to reproduce the results in the last paper of Ref. [5]. To make the statement more conclusive we need more lattice QCD data.

The rest of this paper will be devoted to qualitative explanation of this back-bending curvature as a result of the vector interaction. We will discuss the mechanism in order.

1) We have to realize that the finite $T$ is important to understand the back-bending behavior. The information of the critical temperature is implicit in Figs. 3, 4, and 5. The general tendency is that the critical temperature becomes smaller as $\mu$ increases. Thus, in fact, the critical surface is cut at large chemical potential for which the 


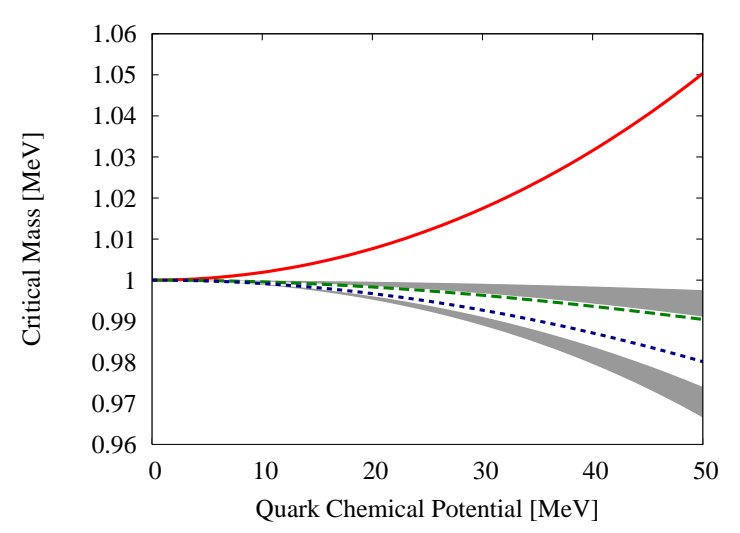

FIG. 6: Critical mass $m_{c}$ along the $m_{u d}=m_{s}$ line as a function of $\mu$. The (red) solid curve represents the PNJL model results at $G_{V}=0$, the (green) dashed curve at $G_{V}=0.4 G_{S}$, and the (blue) dotted curve at $G_{V}=0.8 G_{S}$. The upper and lower shaded regions correspond to the lattice results reported in the first and last papers in Ref. [5], respectively.

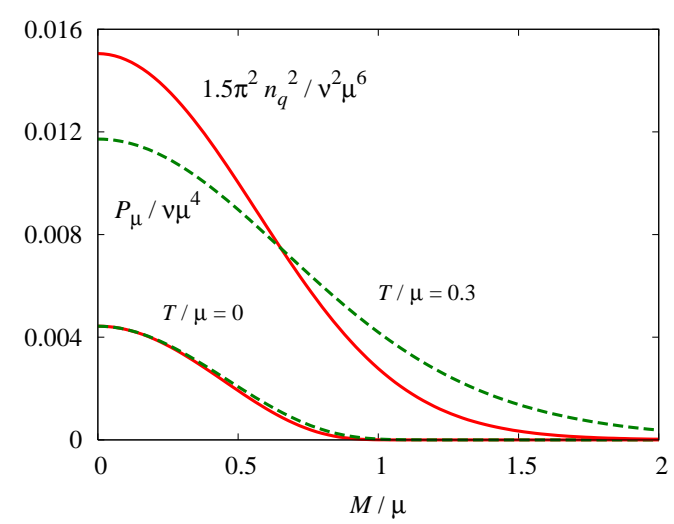

FIG. 7: $n_{q}^{2}$ (solid curve) and $P_{\mu}$ (dotted curve) at $T / \mu=0$ and $T / \mu=0.3$, where $n_{q}^{2}$ is normalized such that $P_{\mu}$ and $n_{q}^{2}$ nearly coincide at $T / \mu=0$.

critical point touches $T=0$.

2) In the vicinity of $\mu=0$, hence, the finite $T$ effect is substantial, which makes the functional forms of the integrated $n_{q}(\mu)$ with respect to $\mu$ (i.e. $P_{\mu}$ ) and $n_{q}^{2}$ deviate from each other unlike the $T=0$ case. It should be mentioned that the simple argument in Sec. III is still applicable for $m_{u d}=m_{s}$ as it is (except for the $\mathrm{U}_{\mathrm{A}}(1)$ breaking term). Then the integrated one (2) turns out to be less steeper than $n_{q}^{2}$ as a function of $M$, which can be readily confirmed by simple numerical calculations (see Fig. (7).

Thus the pressure contribution by density-induced particles is relatively more suppressed by finite $T$ than the vector interaction effect involving $n_{q}^{2}$. This means, in other words, that $n_{q}^{2}$ brings a sharper modification than $P_{\mu}$ to the total pressure at higher $T$ (and thus smaller $\mu$ in turn).

3) Because the repulsive vector interaction has the op- posite effect to the density, as we have seen in Sec. II some $G_{V}$ exists which is large enough to invert the density effect. Still, the first-order region at $\mu=0$ is intact since $n_{q}=0$ at $\mu=0$.

4) With appropriate $G_{V} \neq 0$, as $\mu$ goes up, the firstorder region shrinks by the effect of growing $n_{q}^{2}$ from the vector interaction which overwhelms the effect of $P_{\mu}$ as long as $\mu / T$ is small.

5) When $\mu$ gets larger and $T / \mu$ becomes smaller, the functional shape of $n_{q}^{2}$ comes to be identical to that of $P_{\mu}$ as seen in Fig. 7 . If $G_{V}$ is not too large, the density effect can surpass the vector interaction eventually, which makes the curvature bend back into the standard direction at high $\mu$, which is manifestly the case in Figs. 4 and 5 .

\section{SUMMARY}

We have clarified how the quasi-particle description can lead to a first-order phase transition in cold and dense quark matter. In the same way we have intuitively made clear the role played by the mean-fields from the repulsive vector-vector interaction which reduces a pressure contribution from density-induced particles.

We have then discussed the vector interaction in the $m_{u d}-m_{s}$ plane with three flavors. We have drawn the critical surface using the PNJL model with the vector interaction. Along the same line as the quasi-particle description we have given a simple account for the backbending behavior of the critical surface, which is consistent with the negative curvature in the recent lattice simulations. Logically speaking, therefore, there might be a chance that the critical point still persists even with the negative curvature at small density.

It should be remarked, however, that whether the back bending occurs or not cannot be naively interpreted as whether the critical point exists or not. This is because, as we mentioned, the critical surface is cut at some large $\mu$ where the critical temperature is zero. As a matter of fact, the critical value of $G_{V}$ is around 0.25 as in Eq. (11) (see also Refs. [11, 12, 13]) for which we cannot observe clear back-bending behavior in the PNJL model. In this way, if we take the comparison to the PNJL model analysis seriously, the lattice results by de Forcrand and Philipsen certainly suggest the nonexistence of the QCD critical point.

There are important issues to be investigated in the future. First of all, the determination of $G_{V}$ is indispensable. For this purpose, unfortunately, the description of the vector meson property within the NJL model is not quite reliable because the momentum cutoff is not large enough as compared to the vector meson mass. The lattice simulation is one possibility, though observable to measure $G_{V}$ is not clear yet. Second, not only the vector interaction but also the anomaly ('t Hooft) term is important for the existence of the QCD critical point [7]. The density dependence of the anomaly coupling strength is 
unknown from the lattice simulation, which may affect the critical surface curvature. The density dependence of $G_{V}$ is not known, either, but the point of what we have shown here is that intriguing density dependence appears from $n_{q}^{2}$ even for a constant $G_{V}$. Hence, if the opposite curvature of the critical surface to the standard one is really established in the lattice simulation, it could be interpreted as a circumstantial evidence for finite repulsive vector-vector interaction.

\section{Acknowledgments}

The author is grateful to Teiji Kunihiro and Akira Ohnishi for discussions. He thanks the Institute for $\mathrm{Nu}-$ clear Theory at the University of Washington for its hospitality and the Department of Energy for partial support during the completion of this work. There, he benefited from discussions with Misha Stephanov, Volker Koch, Philippe de Forcrand, Sourendu Gupta, and Maria-Paola Lombardo. He is supported by Japanese MEXT grant No. 20740134 and also supported in part by Yukawa International Program for Quark Hadron Sciences.
[1] M. A. Stephanov, K. Rajagopal and E. V. Shuryak, Phys. Rev. Lett. 81, 4816 (1998); Phys. Rev. D 60, 114028 (1999).

[2] M. Asakawa and K. Yazaki, Nucl. Phys. A 504, 668 (1989); A. Barducci, R. Casalbuoni, S. De Curtis, R. Gatto and G. Pettini, Phys. Lett. B 231, 463 (1989); A. Barducci, R. Casalbuoni, G. Pettini and R. Gatto, Phys. Rev. D 49, 426 (1994).

[3] A. M. Halasz, A. D. Jackson, R. E. Shrock, M. A. Stephanov and J. J. M. Verbaarschot, Phys. Rev. D 58, 096007 (1998).

[4] K. Fukushima, J. Phys. G 35, 104020 (2008).

[5] P. de Forcrand and O. Philipsen, JHEP 0701, 077 (2007); P. de Forcrand, S. Kim and O. Philipsen, PoS LAT2007, 178 (2007); P. de Forcrand and O. Philipsen, arXiv:0808.1096 [hep-lat].

[6] C. Schmidt, PoS LAT2006, 021 arXiv:hep-lat/0610116.

[7] R. D. Pisarski and F. Wilczek, Phys. Rev. D 29, 338 (1984); F. Wilczek, Int. J. Mod. Phys. A 7, 3911 (1992) [Erratum-ibid. A 7, 6951 (1992)].

[8] F. R. Brown et al., Phys. Rev. Lett. 65, 2491 (1990).

[9] S. Gavin, A. Gocksch and R. D. Pisarski, Phys. Rev. D 49, 3079 (1994) arXiv:hep-ph/9311350.

[10] P. Kovacs and Z. Szep, Phys. Rev. D 75, 025015 (2007);
B. J. Schaefer and M. Wagner, arXiv:0808.1491 [hep-ph].

[11] K. Fukushima, Phys. Rev. D 77, 114028 (2008) [Erratum-ibid. D 78, 039902 (2008)].

[12] M. Kitazawa, T. Koide, T. Kunihiro and Y. Nemoto, Prog. Theor. Phys. 108, 929 (2002).

[13] C. Sasaki, B. Friman and K. Redlich, Phys. Rev. D 75, 054026 (2007).

[14] Y. Sakai, K. Kashiwa, H. Kouno, M. Matsuzaki and M. Yahiro, arXiv:0806.4799 [hep-ph].

[15] T. Hatsuda and T. Kunihiro, Phys. Rept. 247, 221 (1994) arXiv:hep-ph/9401310.

[16] A. Gocksch, Phys. Rev. Lett. 67, 1701 (1991).

[17] A. Gocksch and M. Ogilvie, Phys. Rev. D 31, 877 (1985); E. M. Ilgenfritz and J. Kripfganz, Z. Phys. C 29, 79 (1985); K. Fukushima, Phys. Lett. B 591, 277 (2004); C. Ratti, M. A. Thaler and W. Weise, Phys. Rev. D 73, 014019 (2006); K. Kashiwa, H. Kouno, M. Matsuzaki and M. Yahiro, Phys. Lett. B 662, 26 (2008); W. j. Fu, Z. Zhang and Y.x. Liu, Phys. Rev. D 77, 014006 (2008); M. Ciminale, R. Gatto, N. D. Ippolito, G. Nardulli and M. Ruggieri, Phys. Rev. D 77, 054023 (2008).

[18] S. Klimt, M. Lutz and W. Weise, Phys. Lett. B 249, 386 (1990); M. Lutz, S. Klimt and W. Weise, Nucl. Phys. A 542, 521 (1992). 